\title{
A CONTINUITY CORRECTION FOR DISCRETE BARRIER OPTIONS
}

\author{
MARK BROADIE AND PAUL GLASSERMAN
}

Columbia Business School, New York

STEVEN KOU

Department of Statistics, University of Michigan

\begin{abstract}
The payoff of a barrier option depends on whether or not a specified asset price, index, or rate reaches a specified level during the life of the option. Most models for pricing barrier options assume continuous monitoring of the barrier; under this assumption, the option can often be priced in closed form. Many (if not most) real contracts with barrier provisions specify discrete monitoring instants; there are essentially no formulas for pricing these options, and even numerical pricing is difficult. We show, however, that discrete barrier options can be priced with remarkable accuracy using continuous barrier formulas by applying a simple continuity correction to the barrier. The correction shifts the barrier away from the underlying by a factor of $\exp (\beta \sigma \sqrt{\Delta t})$, where $\beta \approx 0.5826, \sigma$ is the underlying volatility, and $\Delta t$ is the time between monitoring instants. The correction is justified both theoretically and experimentally.
\end{abstract}

KEY WORDS: path-dependent options, Siegmund's corrected diffusion approximation, level crossing probabilities

\section{THE MAIN RESULT}

\subsection{Introduction}

A barrier option is activated (knocked in) or extinguished (knocked out) when a specified asset price, index, or rate reaches a specified level. The simplest such options are otherwise standard calls and puts that are knocked in or knocked out by the underlying asset itself. Some variants tie the barrier crossing to one variable and the payoff to another; others specify "binary" payoffs in place of the usual payoffs for calls and puts. Taken together, these are among the most popular options with path-dependent payoffs. The knock-in and knock-out features lower the price of an option, and may provide a payoff distribution that better matches a hedger's risk or a speculator's view.

Most models of barrier options assume continuous monitoring of the barrier: a knock-in or knock-out is presumed to occur if the barrier is breached at any instant in the life of the option. Under this assumption, Merton (1973) obtained a formula for pricing a knock-out call. Subsequent work on pricing continuously monitored barrier options includes Heynen and Kat (1994a, 1994b), Kunitomo and Ikeda (1992), Rich (1994a, 1994b), and Rubinstein and Reiner (1991). However, a sizable portion of real contracts with barrier features specify fixed times for monitoring of the barrier-typically, daily closings. One article in the trade literature (Derivatives Week 1995a) faults existing pricing models for not addressing

Manuscript received September 1995; final revision received April 1996.

Address correspondence to the first two authors at Columbia Business School, 415 Uris Hall, New York, NY 10027; e-mail: mbroadie@ research.gsb.columbia.edu; pglasser@ research.gsb.columbia.edu, or to the third author at the Dept. of Statistics, University of Michigan, Ann Arbor, MI 48109; e-mail: Kou@stat.1sa.umich.edu.

(C) 1997 Blackwell Publishers, 350 Main St., Malden, MA 02148, USA, and 108 Cowley Road, Oxford, OX4 1JF, UK. 
this feature. Another (Derivatives Week 1995b) discusses concerns that when monitoring times are not specified, extraneous barrier breaches may occur in less liquid markets while the major Western markets are closed. Moreover, numerical examples, including those in Chance (1994), Flesaker (1992), and Kat and Verdonk (1995), indicate that there can be substantial price differences between discrete and continuous barrier options, even under daily monitoring of the barrier. Unfortunately, the exact pricing results available for continuous barriers do not extend to the discrete case. ${ }^{1}$ Even numerical methods using standard lattice techniques or Monte Carlo simulation face significant difficulties in incorporating discrete monitoring, as demonstrated in Broadie, Glasserman, and Kou (1996).

In this paper, we introduce a simple continuity correction for approximate pricing of discrete barrier options. Our method uses formulas for the prices of continuous barrier options but shifts the barrier to correct for discrete monitoring. The shift is determined solely by the monitoring frequency, the asset volatility, and a constant $\beta \approx 0.5826$. It is therefore trivial to implement. Compared with using the unadjusted continuous price, our formula reduces the error from $O(1 / \sqrt{m})$ to $o(1 / \sqrt{m})$, as the number of monitoring points $m$ increases. Numerical results indicate that the approximation is accurate enough to correctly price barrier options in all but the most extreme circumstance; i.e., except when the price of the underlying asset nearly coincides with the barrier.

Our analysis is based on the usual Black-Scholes market assumptions (Black and Scholes 1973). In particular, the asset price $\left\{S_{t}, t \geq 0\right\}$ follows the stochastic differential equation

$$
\frac{d S}{S}=v d t+\sigma d Z
$$

where $Z$ is a standard Wiener process, $v$ and $\sigma>0$ are constants, and $S_{0}$ is fixed. The term structure is flat, and we let $r$ denote the constant, continuously compounded risk-free interest rate. The price of a claim contingent on $S$ is the expected present value of its cash flows under the equivalent martingale measure, which sets $v=r$ in (1.1).

Let $H$ denote the level of the barrier. An up option has $H>S_{0}$ and a down option has $H<S_{0}$; in particular, we always assume $H \neq S_{0}$. The asset price reaches the barrier for the first time at ${ }^{2}$

$$
\tau_{H}=\inf \left\{t>0: S_{t}=H\right\}
$$

this is an up-crossing if $S_{0}<H$ and a down-crossing if $S_{0}>H$. A knock-in call option with maturity $T$ and strike $K$ pays $\left(S_{T}-K\right)^{+}$at time $T$ if $\tau_{H} \leq T$ and zero otherwise. Its price is thus ${ }^{3}$

$$
e^{-r T} E\left[\left(S_{T}-K\right)^{+} ; \tau_{H} \leq T\right]
$$

the expectation taken with respect to the equivalent martingale measure. For a put option, replace $\left(S_{T}-K\right)^{+}$with $\left(K-S_{T}\right)^{+}$, and for knock-out options replace the event $\left\{\tau_{H} \leq T\right\}$

\footnotetext{
${ }^{1}$ The price of a discrete barrier option can be expressed in "closed form" in terms of multivariate normal probabilities. The dimension of the relevant multivariate normal distribution is equal to the number of monitoring instants. This is typically too large for numerical evaluation.

${ }^{2}$ Here and in what follows, we adopt the usual convention that the infimum of an empty set is infinity, so that in particular $\tau_{H}=\infty$ if the asset prices never reach the barrier.

${ }^{3}$ For a random variable $X$ and an event $A$, the notation $E[X ; A]$ means $E\left[X \mathbf{1}_{A}\right]$, with $\mathbf{1}_{A}$ the indicator of $A$.
} 
with its complement $\left\{\tau_{H}>T\right\}$. Formulas for pricing these continuously monitored barrier options are reviewed in Section 3.

Now suppose the barrier is monitored only at times $i \Delta t, i=0,1, \ldots, m$, where $\Delta t=$ $T / m$. Let us write $\tilde{S}_{i}$ for $S_{i \Delta t}$, so that $\left\{\tilde{S}_{i}, i=0,1, \ldots\right\}$ is the asset price at monitoring instants. Define

$$
\tilde{\tau}_{H}= \begin{cases}\inf \left\{n>0: \tilde{S}_{n}>H\right\}, & S_{0}<H \\ \inf \left\{n>0: \tilde{S}_{n}<H\right\}, & S_{0}>H\end{cases}
$$

The price of a discrete knock-in call option is given by

$$
e^{-r T} E\left[\left(\tilde{S}_{m}-K\right)^{+} ; \tilde{\tau}_{H} \leq m\right]
$$

the same modifications as before yield puts and knock-outs.

In general, there are no easily computed closed-form expressions for the prices of these discrete barrier options. A consequence of our analysis is the obvious conclusion that the discrete price converges to the continuous price as the monitoring frequency increases, suggesting that the continuous price may be used as a naive approximation. The following result shows how to adjust the continuous formula to obtain a far better approximation to the discrete price.

THEOREM 1.1. Let $V_{m}(H)$ be the price of a discretely monitored knock-in or knockout down call or up put with barrier $H$. Let $V(H)$ be the price of the corresponding continuously monitored barrier option. Then

$$
V_{m}(H)=V\left(H e^{ \pm \beta \sigma \sqrt{T / m}}\right)+o\left(\frac{1}{\sqrt{m}}\right)
$$

where + applies if $H>S_{0}$, - applies if $H<S_{0}$, and $\beta=-\zeta\left(\frac{1}{2}\right) / \sqrt{2 \pi} \approx 0.5826$, with $\zeta$ the Riemann zeta function.

This result indicates that to use the continuous price as an approximation to the discrete price, we should first shift the barrier away from $S_{0}$ by a factor of $\exp (\beta \sigma \sqrt{\Delta t})$. Numerical results in Section 2 suggest that the resulting approximation is remarkably accurate.

\subsection{A Sketch of the Argument}

Sections 3 and 4 and the appendices are devoted to the proof of Theorem 1.1. To give some insight into the result, we illustrate the argument for an example. Consider a binary knock-in option paying one dollar if the asset price reaches $H$ at some point in $[0, T]$ and if at time $T$ it is below $K$, with $H>\max \left\{S_{0}, K\right\}$. A by-product of the analysis in later sections (Corollary 4.1) is that Theorem 1.1 applies to binary barrier options as well. Suppose, for purposes of illustration, that $r-\frac{1}{2} \sigma^{2}=0$, so that under the equivalent martingale measure $\log S_{t}$ has zero drift. ${ }^{4}$ Define $b$ and $c$ via the relations $H=S_{0} \exp (b \sigma \sqrt{T})$ and

\footnotetext{
${ }^{4}$ Ito's lemma applied to (1.1) yields $d \log S=\left(v-\frac{1}{2} \sigma^{2}\right) d t+\sigma d Z$.
} 
$K=S_{0} \exp (c \sigma \sqrt{T})$. Assuming continuous monitoring of the barrier, the value of the option is $e^{-r T}$ times

$$
\begin{aligned}
P\left(S_{T}<K, \tau_{H} \leq T\right) & =P\left(\log S_{T}<c \sigma \sqrt{T}, \max _{0 \leq t \leq T} \log S_{t} \geq b \sigma \sqrt{T}\right) \\
& =P\left(\log S_{T} \geq(2 b-c) \sigma \sqrt{T}\right) \\
& =1-\Phi(2 b-c),
\end{aligned}
$$

where $\Phi$ is the standard normal cumulative distribution function. Equation (1.4) is an application of the reflection principle (Karatzas and Shreve 1991, pp. 79-80).

Suppose, now, that for the option to be knocked in, the asset price must reach $H$ at some time in $\{0, \Delta t, 2 \Delta t, \ldots, m \Delta t\}$, with $\Delta t=T / m$. Because $r-\frac{1}{2} \sigma^{2}=0$, we may represent the asset price at these monitoring instants as

$$
\tilde{S}_{n}=S_{0} e^{\sigma \sqrt{\Delta t} \tilde{W}_{n}}, \quad \text { with } \tilde{W}_{n}=\sum_{i=1}^{n} Z_{i}
$$

where the $Z_{i}$ are independent standard normal random variables. The price of the option under discrete monitoring of the barrier is $e^{-r T}$ times

$$
P\left(\tilde{S}_{m}<K, \max _{0 \leq n \leq m} \tilde{S}_{n} \geq H\right)=P\left(\tilde{W}_{m}<c \sqrt{m}, \tilde{\tau} \leq m\right)
$$

where $\tilde{\tau}$ is the first time $\tilde{W}$ exceeds $b \sqrt{m}$. The increments of the random walk $\tilde{W}$ are symmetrically distributed, so the reflection principle yields

$$
P\left(\tilde{W}_{m}<c \sqrt{m}, \tilde{\tau} \leq m\right)=P\left(\tilde{W}_{m}>2\left(b \sqrt{m}+R_{m}\right)-c \sqrt{m}\right)
$$

where $R_{m} \triangleq \tilde{W}_{\tilde{\tau}}-b \sqrt{m}$ is the overshoot above level $b \sqrt{m}$ (see Figure 1.1). Notice that $P\left(\tilde{W}_{m}>x \sqrt{m}\right)=1-\Phi(x)$. Applying this to (1.6) and treating $\tilde{W}_{m}$ and $R_{m}$ as though they were independent we get

$$
P\left(\tilde{W}_{m}>2\left(b \sqrt{m}+R_{m}\right)-c \sqrt{m}\right) \approx E\left[1-\Phi\left(2\left(b+\frac{R_{m}}{\sqrt{m}}\right)-c\right)\right] .
$$

Expanding in a formal Taylor series with $\varphi$ denoting the standard normal density, and using the fact (to be reviewed in Section 4.3) that $E\left[R_{m}\right] \rightarrow \beta$, we get

$$
\begin{aligned}
P\left(\tilde{W}_{m}>2\left(b \sqrt{m}+R_{m}\right)-c \sqrt{m}\right) & \approx E\left[1-\Phi(2 b-c)-\frac{2 R_{m}}{\sqrt{m}} \varphi(2 b-c)+o\left(\frac{1}{\sqrt{m}}\right)\right] \\
& \approx 1-\Phi(2 b-c)-\frac{2}{\sqrt{m}} E\left[R_{m}\right] \varphi(2 b-c)+o\left(\frac{1}{\sqrt{m}}\right)
\end{aligned}
$$




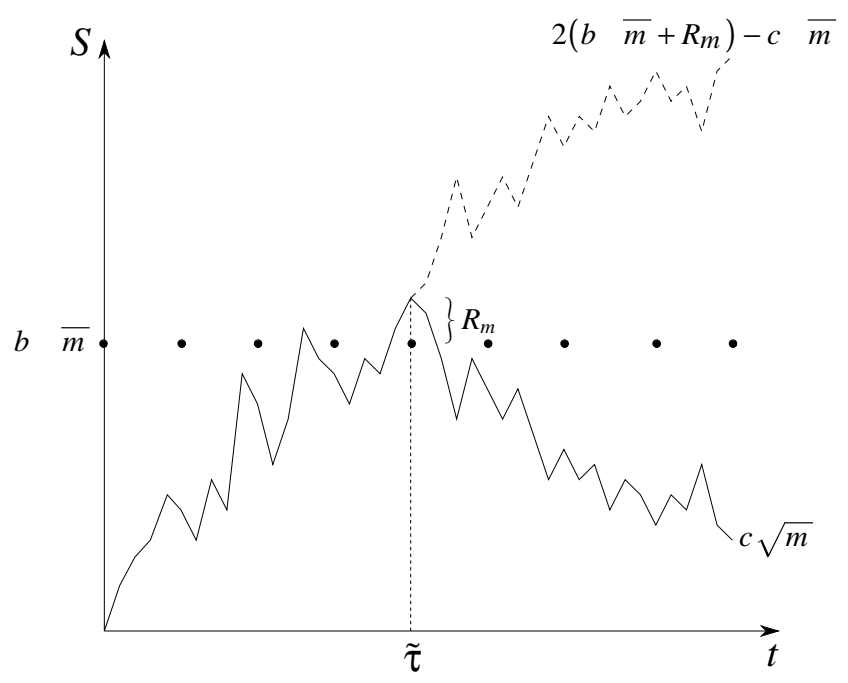

FIGURE 1.1. Overshoot and reflection principle illustration.

$$
\begin{aligned}
& =1-\Phi(2 b-c)-\frac{2 \beta}{\sqrt{m}} \varphi(2 b-c)+o\left(\frac{1}{\sqrt{m}}\right) \\
& =1-\Phi\left(2\left(b+\frac{\beta}{\sqrt{m}}\right)-c\right)+o\left(\frac{1}{\sqrt{m}}\right) .
\end{aligned}
$$

In light of (1.5), this is

$$
P\left(S_{T}<K, \tau_{H \exp (\beta \sigma \sqrt{\Delta t})} \leq T\right)+o\left(\frac{1}{\sqrt{m}}\right)
$$

and thus agrees, up to $o(1 / \sqrt{m})$, with the price of a continuously monitored barrier option knocked in at level $H \exp (\beta \sigma \sqrt{\Delta t})$. The difference $H e^{\beta \sigma \sqrt{\Delta t}}-H$ may be viewed as an approximation to the amount by which the asset price exceeds the barrier at the first monitoring instant at which it is above the barrier.

The heuristic argument just sketched points out the key steps in proving Theorem 1.1 rigorously: we find a suitable representation of the discrete price, expand it in a Taylor series, evaluate the limits of the coefficients as $m \rightarrow \infty$, and identify the resulting expression as an expansion of the continuous price. Evaluation of the limits of coefficients requires consideration of the joint limit of the overshoot and the barrier-crossing time; the necessary asymptotic independence result is given in Section 4.3, and this may be viewed as the rigorous counterpart of (1.7). Handling ordinary barrier options instead of just binary options requires consideration of integrals of the probabilities outlined above.

Continuity corrections for level-crossing probabilities of random walks have a long history in sequential analysis and, to a lesser extent, in risk theory. Key references from the statistical literature are Chernoff (1965), Siegmund (1979, 1985), Siegmund and Yuh 
(1982), and Woodroofe (1982); for developments in risk theory see Asmussen (1989). We especially build on the work of Siegmund and Yuh. Also in the context of sequential analysis, Chernoff and Petkau (1986) have shown that a boundary correction improves the numerical solution of an optimal stopping problem based on a binomial approximation to a diffusion. Recently, AitSahlia (1995) has analyzed a similar technique in the pricing of American options (without barriers).

\section{PERFORMANCE OF THE CORRECTED BARRIER APPROXIMATION}

Before proceeding with the main proof, we present numerical results to indicate the accuracy of the corrected barrier approximation given in equation (1.3). Table 2.1 compares the approximation (and two related approximations to be described shortly) with the true value for different levels of the barrier $H$. The options considered are down-and-out call options, i.e., $S_{0}>H$, and the option becomes worthless if $S$ crosses the barrier before the option expires at time $T$. The "true" value is determined from a trinomial procedure modified in several ways to specifically handle discrete barriers and is fully described in Broadie et al. (1996). This numerical procedure has an average accuracy of about 0.001 for this range of parameters.

Table 2.1 and later tables show that the continuous barrier price can differ from the discrete barrier price by economically significant amounts. For example, for $H=97$ in Table 2.1, the continuous barrier price is $\$ 3.06$ while the discrete barrier option with daily monitoring is worth $25 \%$ more (\$3.83). The prices under approximation (1) in the tables result from the corrected continuous barrier formula suggested by Theorem 1.1. The relative absolute error of this formula is less than $0.1 \%$ for $H \leq 98$; overall, this formula is remarkably accurate except in extreme cases with $H$ very close to $S_{0}$. It can be evaluated very fast (more than $10,000 / \mathrm{sec}$ on an Intel Pentium $133 \mathrm{MHz}$ processor), while the procedure used to compute the true value to comparable accuracy is many orders of magnitude slower (approximately one hour on the same processor). ${ }^{5}$

Table 2.1 shows results for two other approximations. Approximation (2) uses a formula of Heynen and Kat (1994b) for continuous partial barrier options, i.e., options whose barriers are monitored throughout a time interval $\left[0, t_{1}\right]$ or $\left[t_{1}, T\right]$, with $0<t_{1}<T$. The first monitoring point for a discrete barrier option does not occur until $\Delta t$, suggesting an approximation that shifts the barrier in the formula for a partial barrier option monitored during $[\Delta t, T]$. Specifically, approximation (2) sets $t_{1}=\Delta t$ and shifts the barrier in equation (9) on p. 259 of Heynen and Kat (1994b). It can be evaluated in about the same (negligible) computing time as the first approximation. Although correcting the continuous barrier formula (approximation (1)) leads to small underpricing in some cases, the corrected partial barrier formula (approximation (2)) leads to small overpricing in these cases.

The modified corrected partial barrier approximation (3) in Table 2.1 is a further heuristic that attempts to compromise between the slight underpricing and overpricing observed with (1) and (2). It does so by adjusting the beginning of the partial barrier to $t_{1}=0.58 \Delta t$. (The factor 0.58 was found experimentally to be the best among a few different values tried.) In most of the test cases, this heuristic had the smallest error of the three approximations. It is worth emphasizing, however, that only approximation (1) is rigorously supported; an attempt to justify a refinement of (1) would involve the daunting task of carrying out the

\footnotetext{
${ }^{5}$ In order to compute the true value to within 0.001 on average, we needed to run a modified trinomial routine using 80,000 time steps. The large number of steps required for this accuracy is quite computationally intensive.
} 
TABLE 2.1

Down-and-Out Call Option Price Results, $m=50$ barrier points

\begin{tabular}{cccccccccr}
\hline & \multicolumn{3}{c}{$\begin{array}{c}\text { Corrected } \\
\text { continuous } \\
\text { barrier }\end{array}$} & $\begin{array}{c}\text { Corrected } \\
\text { partial } \\
\text { barrier }\end{array}$ & $\begin{array}{c}\text { Modified } \\
\text { corrected } \\
\text { partial }\end{array}$ & \multicolumn{3}{c}{$\begin{array}{c}\text { Relative error } \\
\text { (in percent) }\end{array}$} \\
\hline 85 & $\begin{array}{c}\text { Continuous } \\
\text { barrier }\end{array}$ & $(1)$ & $(2)$ & $(3)$ & True & $(1)$ & $(2)$ & $(3)$ \\
\hline 86 & 6.308 & 6.322 & 6.322 & 6.322 & 6.322 & 0.0 & 0.0 & 0.0 \\
87 & 6.244 & 6.306 & 6.306 & 6.306 & 6.306 & 0.0 & 0.0 & 0.0 \\
88 & 6.185 & 6.281 & 6.281 & 6.281 & 6.281 & 0.0 & 0.0 & 0.0 \\
89 & 6.099 & 6.184 & 6.242 & 6.242 & 6.242 & 0.0 & 0.0 & 0.0 \\
90 & 5.977 & 6.098 & 6.098 & 6.098 & 6.098 & 0.0 & 0.0 & 0.0 \\
91 & 5.808 & 5.977 & 5.977 & 5.977 & 5.977 & 0.0 & 0.0 & 0.0 \\
92 & 5.579 & 5.810 & 5.810 & 5.810 & 5.810 & 0.0 & 0.0 & 0.0 \\
93 & 5.277 & 5.585 & 5.585 & 5.585 & 5.584 & 0.0 & 0.0 & 0.0 \\
94 & 4.888 & 5.288 & 5.288 & 5.288 & 5.288 & 0.0 & 0.0 & 0.0 \\
95 & 4.398 & 4.907 & 4.907 & 4.907 & 4.907 & 0.0 & 0.0 & 0.0 \\
96 & 3.792 & 4.428 & 4.429 & 4.428 & 4.427 & 0.0 & 0.1 & 0.0 \\
97 & 3.060 & 3.836 & 3.845 & 3.837 & 3.834 & 0.1 & 0.3 & 0.1 \\
98 & 2.189 & 3.121 & 3.160 & 3.129 & 3.126 & -0.2 & 1.1 & 0.1 \\
99 & 1.171 & 2.271 & 2.408 & 2.321 & 2.337 & -2.8 & 3.0 & -0.6 \\
\hline
\end{tabular}

Option parameters: $S_{0}=100, K=100, \sigma=0.30, r=0.10$, and $T=0.2$. Assuming 250 trading days per year, $m=50$ barrier points roughly corresponds to daily monitoring of the barrier.

expansions in Sections 3 and 4 to one more term. Also, approximations (2) and (3) are slightly more complicated to implement than approximation (1).

Table 2.2 shows that the approximations degrade slowly as the number of monitoring points decreases. Indeed, even though Theorem 1.1 is based on the limit as $m$ increases to infinity, the approximation is quite accurate even for $m=5$, in marked contrast to the continuous price. Table 2.3 shows results for high asset volatility $(\sigma=0.6)$, long maturity $(T=2)$, and higher strike $(K=110)$. The overall performance of the approximations remains excellent, except when the barrier is very close to the underlying asset price. In this case, the relative errors are slightly larger with higher volatility and longer time to maturity and are slightly lower with a higher strike (compared to Table 2.1).

Option price derivatives (e.g., "delta" $=\partial V / \partial S_{0}$ ) are of particular importance to practitioners for hedging and risk management. Although this case is not covered by Theorem 1.1, the correction can be applied to the continuous delta formula as well. Table 2.4 shows how the deltas of the three approximations compare to the true delta (determined through extensive numerical computations). Relative absolute errors are less than $0.1 \%$ for $H \leq 97$ for approximation (1). Delta approximations (2) and (3) are better than (1) for $H$ very close to $S_{0}$.

Next we consider a two-state example where the payoff of the option is determined by one state variable and the knock in and or knock out is determined by a second state variablefor example, options on stocks or stock indices which are knocked out if a currency reaches a barrier trade in the over-the-counter market. Rich (1996) models options with default risk in this framework. Heynan and Kat (1994a) and Rich (1996) derive formulas for such 
TABLE 2.2

Down-and-Out Call Option Price Results, varying $m$

\begin{tabular}{|c|c|c|c|c|c|c|c|c|c|}
\hline \multirow[b]{2}{*}{$m$} & \multirow[b]{2}{*}{ Barrier } & \multirow{2}{*}{$\begin{array}{c}\text { Continuous } \\
\text { barrier }\end{array}$} & \multirow{2}{*}{$\begin{array}{l}\text { Corrected } \\
\text { continuous } \\
\text { barrier } \\
\text { (1) }\end{array}$} & \multirow{2}{*}{$\begin{array}{l}\text { Corrected } \\
\text { partial } \\
\text { barrier } \\
(2)\end{array}$} & \multirow{2}{*}{$\begin{array}{l}\text { Modified } \\
\text { corrected } \\
\text { partial } \\
\text { (3) }\end{array}$} & \multirow[b]{2}{*}{ True } & \multicolumn{3}{|c|}{$\begin{array}{l}\text { Relative error } \\
\text { (in percent) }\end{array}$} \\
\hline & & & & & & & (1) & (2) & (3) \\
\hline \multirow{8}{*}{25} & 85 & 6.308 & 6.327 & 6.327 & 6.327 & 6.326 & 0.0 & 0.0 & 0.0 \\
\hline & 87 & 6.244 & 6.293 & 6.293 & 6.293 & 6.292 & 0.0 & 0.0 & 0.0 \\
\hline & 89 & 6.099 & 6.210 & 6.210 & 6.210 & 6.210 & 0.0 & 0.0 & 0.0 \\
\hline & 91 & 5.808 & 6.033 & 6.033 & 6.033 & 6.032 & 0.0 & 0.0 & 0.0 \\
\hline & 93 & 5.277 & 5.688 & 5.688 & 5.688 & 5.688 & 0.0 & 0.0 & 0.0 \\
\hline & 95 & 4.398 & 5.084 & 5.088 & 5.084 & 5.081 & 0.0 & 0.1 & 0.0 \\
\hline & 97 & 3.060 & 4.113 & 4.154 & 4.120 & 4.116 & -0.1 & 0.9 & 0.1 \\
\hline & 99 & 1.171 & 2.673 & 2.923 & 2.779 & 2.813 & -5.0 & 3.9 & -1.2 \\
\hline \multirow{8}{*}{5} & 85 & 6.308 & 6.337 & 6.338 & 6.337 & 6.337 & 0.0 & 0.0 & 0.0 \\
\hline & 87 & 6.244 & 6.323 & 6.323 & 6.323 & 6.321 & 0.0 & 0.0 & 0.0 \\
\hline & 89 & 6.099 & 6.284 & 6.287 & 6.285 & 6.281 & 0.1 & 0.1 & 0.1 \\
\hline & 91 & 5.808 & 6.194 & 6.205 & 6.195 & 6.187 & 0.1 & 0.3 & 0.1 \\
\hline & 93 & 5.277 & 6.004 & 6.043 & 6.011 & 6.000 & 0.1 & 0.7 & 0.2 \\
\hline & 95 & 4.398 & 5.646 & 5.760 & 5.676 & 5.671 & -0.5 & 1.6 & 0.1 \\
\hline & 97 & 3.060 & 5.028 & 5.323 & 5.141 & 5.167 & -2.7 & 3.0 & -0.5 \\
\hline & 99 & 1.171 & 4.050 & 4.724 & 4.392 & 4.489 & -9.8 & 5.2 & -2.2 \\
\hline
\end{tabular}

Option parameters: $S_{0}=100, K=100, \sigma=0.30, r=0.10$, and $T=0.2$.

two-state options when the barrier is continuously monitored. More specifically, suppose that the risk-neutralized asset price processes are $d S^{i}=S^{i}\left(r d t+\sigma_{i} d Z_{i}\right), i=1,2$, where $Z_{1}$ and $Z_{2}$ have a constant correlation $\rho$. Table 2.5 presents results for a two-asset discrete barrier down-and-out call option. The payoff of the option is $\left(S_{T}^{1}-K\right)^{+}$if $S^{2}$ is greater than $H$ at all monitoring times. The "true" price is more difficult to determine because of the extra dimension involved, but the two-dimensional trinomial procedure used has an average accuracy of about 0.003 for this range of parameters. Table 2.5 shows that the corrected continuous barrier formula is very accurate as long as the barrier is not too close to the initial price of asset 2 .

The previous examples involved down-call options, i.e., options that are knocked out when an asset price decreases to a barrier level, but whose payoff is positive if the terminal asset price is above a strike level. Table 2.6 gives results for up call options (not covered by Theorem 1.1). The results indicate that the corrected barrier formula works well for these options, though perhaps not as well as for down calls. Corrected versions of the continuous partial barrier formulas in Heynen and Kat (1994b) could also be used to approximate the discrete barrier price.

\section{THE CONTINUOUS PRICE}

The purpose of this section is to derive expressions for the continuous price $V(H)$ and its derivative. In this and the remaining sections, we detail the case of the down-and-in call, 
TABLE 2.3

Down-and-Out Call Option Price Results, varying $\sigma, T$, and $K$

\begin{tabular}{|c|c|c|c|c|c|c|c|c|c|}
\hline \multirow[b]{2}{*}{ Panel } & \multirow[b]{2}{*}{ Barrier } & \multirow{2}{*}{$\begin{array}{c}\text { Continuous } \\
\text { barrier }\end{array}$} & \multirow{2}{*}{$\begin{array}{l}\text { Corrected } \\
\text { continuous } \\
\text { barrier } \\
\text { (1) }\end{array}$} & \multirow{2}{*}{$\begin{array}{c}\text { Corrected } \\
\text { partial } \\
\text { barrier } \\
\text { (2) }\end{array}$} & \multirow{2}{*}{$\begin{array}{c}\text { Modified } \\
\text { corrected } \\
\text { partial } \\
\text { (3) }\end{array}$} & \multirow[b]{2}{*}{ True } & \multicolumn{3}{|c|}{$\begin{array}{l}\text { Relative error } \\
\text { (in percent) }\end{array}$} \\
\hline & & & & & & & (1) & (2) & (3) \\
\hline \multirow{8}{*}{ A } & 85 & 10.048 & 10.505 & 10.505 & 10.505 & 10.505 & 0.0 & 0.0 & 0.0 \\
\hline & 87 & 9.404 & 10.020 & 10.020 & 10.020 & 10.020 & 0.0 & 0.0 & 0.0 \\
\hline & 89 & 8.578 & 9.384 & 9.384 & 9.384 & 9.383 & 0.0 & 0.0 & 0.0 \\
\hline & 91 & 7.547 & 8.573 & 8.574 & 8.573 & 8.572 & 0.0 & 0.0 & 0.0 \\
\hline & 93 & 6.293 & 7.566 & 7.572 & 7.566 & 7.563 & 0.0 & 0.1 & 0.0 \\
\hline & 95 & 4.803 & 6.346 & 6.379 & 6.351 & 6.344 & 0.0 & 0.6 & 0.1 \\
\hline & 97 & 3.067 & 4.900 & 5.033 & 4.937 & 4.941 & -0.8 & 1.9 & -0.1 \\
\hline & 99 & 1.084 & 3.219 & 3.638 & 3.414 & 3.475 & -7.4 & 4.7 & -1.8 \\
\hline \multirow{8}{*}{ B } & 85 & 18.856 & 20.821 & 20.822 & 20.821 & 20.819 & 0.0 & 0.0 & 0.0 \\
\hline & 87 & 17.232 & 19.573 & 19.577 & 19.574 & 19.571 & 0.0 & 0.0 & 0.0 \\
\hline & 89 & 15.361 & 18.120 & 18.133 & 18.121 & 18.114 & 0.0 & 0.1 & 0.0 \\
\hline & 91 & 13.227 & 16.446 & 16.487 & 16.450 & 16.436 & 0.1 & 0.3 & 0.1 \\
\hline & 93 & 10.815 & 14.534 & 14.650 & 14.553 & 14.537 & 0.0 & 0.8 & 0.1 \\
\hline & 95 & 8.111 & 12.371 & 12.657 & 12.446 & 12.451 & -0.6 & 1.7 & 0.0 \\
\hline & 97 & 5.104 & 9.945 & 10.576 & 10.183 & 10.254 & -3.0 & 3.1 & -0.7 \\
\hline & 99 & 1.782 & 7.243 & 8.499 & 7.874 & 8.061 & -10.1 & 5.4 & -2.3 \\
\hline \multirow{8}{*}{$\mathrm{C}$} & 85 & 2.494 & 2.496 & 2.496 & 2.496 & 2.496 & 0.0 & 0.0 & 0.0 \\
\hline & 87 & 2.485 & 2.491 & 2.491 & 2.491 & 2.491 & 0.0 & 0.0 & 0.0 \\
\hline & 89 & 2.459 & 2.475 & 2.475 & 2.475 & 2.475 & 0.0 & 0.0 & 0.0 \\
\hline & 91 & 2.394 & 2.433 & 2.433 & 2.433 & 2.433 & 0.0 & 0.0 & 0.0 \\
\hline & 93 & 2.250 & 2.336 & 2.337 & 2.337 & 2.336 & 0.0 & 0.0 & 0.0 \\
\hline & 95 & 1.964 & 2.136 & 2.136 & 2.136 & 2.135 & 0.0 & 0.0 & 0.0 \\
\hline & 97 & 1.446 & 1.757 & 1.761 & 1.757 & 1.756 & 0.1 & 0.3 & 0.1 \\
\hline & 99 & 0.591 & 1.105 & 1.171 & 1.129 & 1.136 & -2.7 & 3.0 & -0.6 \\
\hline
\end{tabular}

Option parameters: $S_{0}=100$ and $r=0.10$. There are $m=50$ barrier points (daily monitoring). Panel A has $\sigma=0.6, K=100, T=0.2$; Panel B has $T=2.0, \sigma=0.3, K=100$; and Panel $\mathrm{C}$ has $K=110, \sigma=0.3$, $T=0.2$.

the other cases following with only minor modifications. Thus,

$$
V(H)=e^{-r T} E\left[\left(S_{T}-K\right)^{+} ; \tau_{H}<T\right]
$$

with $H<S_{0}$ and $H<K$. We define $W$ to be a Wiener process with variance parameter $\sigma^{2}$ and drift $\mu \triangleq r-\frac{1}{2} \sigma^{2}$. This allows us to represent the asset price as $S_{t}=S_{0} \exp \left(W_{t}\right)$ and then work with $W$ rather than $S$. The barrier is breached when $W$ hits $a \triangleq \log \left(H / S_{0}\right)$; i.e.,

$$
\begin{aligned}
\tau_{H} & =\inf \left\{t \geq 0: S_{0} e^{W_{t}} \leq H\right\} \\
& =\inf \left\{t \geq 0: W_{t} \leq a\right\} .
\end{aligned}
$$


TABLE 2.4

Down-and-Out Call Option Delta Results, $m=50$ barrier points

\begin{tabular}{|c|c|c|c|c|c|c|c|c|}
\hline \multirow[b]{2}{*}{ Barrier } & \multirow{2}{*}{$\begin{array}{c}\text { Continuous } \\
\text { barrier }\end{array}$} & \multirow{2}{*}{$\begin{array}{l}\text { Corrected } \\
\text { continuous } \\
\text { barrier } \\
\text { (1) }\end{array}$} & \multirow{2}{*}{$\begin{array}{c}\text { Corrected } \\
\text { partial } \\
\text { barrier } \\
\text { (2) }\end{array}$} & \multirow{2}{*}{$\begin{array}{c}\text { Modified } \\
\text { corrected } \\
\text { partial } \\
\text { (3) }\end{array}$} & \multirow[b]{2}{*}{ True } & \multicolumn{3}{|c|}{$\begin{array}{l}\text { Relative error } \\
\text { (in percent) }\end{array}$} \\
\hline & & & & & & (1) & (2) & (3) \\
\hline 85 & 0.594 & 0.591 & 0.591 & 0.591 & 0.591 & 0.0 & 0.0 & 0.0 \\
\hline 86 & 0.599 & 0.594 & 0.594 & 0.594 & 0.594 & 0.0 & 0.0 & 0.0 \\
\hline 87 & 0.607 & 0.600 & 0.600 & 0.600 & 0.600 & 0.0 & 0.0 & 0.0 \\
\hline 88 & 0.618 & 0.607 & 0.607 & 0.607 & 0.607 & 0.0 & 0.0 & 0.0 \\
\hline 89 & 0.633 & 0.618 & 0.618 & 0.618 & 0.618 & 0.0 & 0.0 & 0.0 \\
\hline 90 & 0.653 & 0.633 & 0.633 & 0.633 & 0.633 & 0.0 & 0.0 & 0.0 \\
\hline 91 & 0.679 & 0.653 & 0.653 & 0.653 & 0.653 & 0.0 & 0.0 & 0.0 \\
\hline 92 & 0.711 & 0.678 & 0.678 & 0.678 & 0.678 & 0.0 & 0.0 & 0.0 \\
\hline 93 & 0.752 & 0.710 & 0.710 & 0.710 & 0.711 & 0.0 & 0.0 & 0.0 \\
\hline 94 & 0.800 & 0.750 & 0.750 & 0.750 & 0.750 & 0.0 & 0.0 & 0.0 \\
\hline 95 & 0.857 & 0.798 & 0.797 & 0.798 & 0.798 & 0.0 & -0.1 & 0.0 \\
\hline 96 & 0.921 & 0.853 & 0.851 & 0.853 & 0.854 & -0.1 & -0.4 & -0.2 \\
\hline 97 & 0.994 & 0.917 & 0.904 & 0.915 & 0.917 & 0.0 & -1.4 & -0.2 \\
\hline 98 & 1.073 & 0.988 & 0.938 & 0.972 & 0.966 & 2.2 & -2.9 & 0.6 \\
\hline 99 & 1.158 & 1.066 & 0.922 & 0.988 & 0.958 & 11.3 & -3.7 & 3.1 \\
\hline
\end{tabular}

Option parameters: $S_{0}=100, K=100, \sigma=0.30, r=0.10$, and $T=0.2$. There are $m=50$ barrier points (daily monitoring).

Define

$$
\operatorname{BSC}(s)=e^{-r T} E\left[\left(s e^{W_{T}}-K\right)^{+}\right]
$$

the Black-Scholes price of a call option as a function of the initial asset price. Then we have

Proposition 3.1. For a down-and-in call,

$$
\begin{aligned}
V(H)= & \left(\frac{H}{S_{0}}\right)^{\left(2 r / \sigma^{2}\right)-1} \operatorname{BSC}\left(H^{2} / S_{0}\right) \\
= & e^{2 \mu a / \sigma^{2}} \operatorname{BSC}\left(S_{0} e^{2 a}\right) \\
= & e^{-r T} e^{2 \mu a / \sigma^{2}} \int_{\left[\log \left(K / S_{0}\right)\right] / \sigma \sqrt{T}}^{\infty}\left(1-\Phi\left(x-\frac{\mu \sqrt{T}}{\sigma}-\frac{2 a}{\sigma \sqrt{T}}\right)\right) \\
& \times S_{0} e^{x \sigma \sqrt{T}} \sigma \sqrt{T} d x .
\end{aligned}
$$

Proof. The first identity is a consequence of Merton's (1973, eqn. (55)) formula for a barrier option; this particular representation is from Boyle and Lau (1994). The second identity restates the first because $a=\log \left(H / S_{0}\right)$. To get the last identity, write the Black- 
TABLE 2.5

Two-Asset Down-and-Out Call Option Price Results, $m=50$ barrier points

\begin{tabular}{|c|c|c|c|c|}
\hline Barrier & $\begin{array}{c}\text { Continuous } \\
\text { barrier }\end{array}$ & $\begin{array}{l}\text { Corrected } \\
\text { continuous } \\
\text { barrier }\end{array}$ & True & $\begin{array}{c}\text { Relative } \\
\text { error } \\
\text { (in percent) }\end{array}$ \\
\hline 85 & 5.893 & 5.986 & 5.985 & 0.0 \\
\hline 86 & 5.772 & 5.887 & 5.886 & 0.0 \\
\hline 87 & 5.626 & 5.766 & 5.765 & 0.0 \\
\hline 88 & 5.452 & 5.621 & 5.620 & 0.0 \\
\hline 89 & 5.246 & 5.448 & 5.447 & 0.0 \\
\hline 90 & 5.005 & 5.244 & 5.243 & 0.0 \\
\hline 91 & 4.726 & 5.005 & 5.005 & 0.0 \\
\hline 92 & 4.404 & 4.729 & 4.729 & 0.0 \\
\hline 93 & 4.038 & 4.412 & 4.413 & 0.0 \\
\hline 94 & 3.623 & 4.051 & 4.052 & 0.0 \\
\hline 95 & 3.158 & 3.643 & 3.645 & -0.1 \\
\hline 96 & 2.639 & 3.185 & 3.187 & -0.1 \\
\hline 97 & 2.066 & 2.676 & 2.679 & -0.1 \\
\hline 98 & 1.435 & 2.112 & 2.121 & -0.4 \\
\hline 99 & 0.747 & 1.493 & 1.542 & -3.2 \\
\hline
\end{tabular}

Option parameters: Initial assets prices are $S^{1}=S^{2}=100, K=100$, $\sigma_{1}=\sigma_{2}=0.30, r=0.10, \rho=0.5$, and $T=0.2$. There are $m=50$ barrier points (daily monitoring). The option is knocked out if $S^{2}$ hits the barrier at a monitoring time. The option payoff is $\left(S_{T}^{1}-K\right)^{+}$at time $T$ if the option has not been knocked out.

TABLE 2.6

Up-and-Out Call Option Price Results, $m=50$ barrier points

\begin{tabular}{ccccc}
\hline Barrier & $\begin{array}{c}\text { Continuous } \\
\text { barrier }\end{array}$ & $\begin{array}{c}\text { Corrected } \\
\text { continuous } \\
\text { barrier }\end{array}$ & \multicolumn{1}{c}{ True } & $\begin{array}{c}\text { Relative } \\
\text { error } \\
\text { (in percent) }\end{array}$ \\
\hline 155 & 12.775 & 12.905 & 12.894 & 0.1 \\
150 & 12.240 & 12.448 & 12.431 & 0.1 \\
145 & 11.395 & 11.707 & 11.684 & 0.2 \\
140 & 10.144 & 10.581 & 10.551 & 0.3 \\
135 & 8.433 & 8.994 & 8.959 & 0.4 \\
130 & 6.314 & 6.959 & 6.922 & 0.5 \\
125 & 4.012 & 4.649 & 4.616 & 0.7 \\
120 & 1.938 & 2.442 & 2.418 & 1.0 \\
115 & 0.545 & 0.819 & 0.807 & 1.5 \\
\hline
\end{tabular}

Option parameters: $S_{0}=110, K=100, \sigma=0.30, r=0.10$, and $T=0.2$. 
Scholes price as

$$
\begin{aligned}
\operatorname{BSC}(s) & =e^{-r T} E\left[\left(s e^{W_{T}}-K\right)^{+}\right] \\
& =e^{-r T} \int_{-\infty}^{\infty}\left(s e^{y}-K\right)^{+} \frac{1}{\sigma \sqrt{T}} \varphi\left(\frac{y-\mu T}{\sigma \sqrt{T}}\right) d y \\
& =e^{-r T} \int_{\log (K / s)}^{\infty}\left(1-\Phi\left(\frac{y-\mu T}{\sigma \sqrt{T}}\right)\right) s e^{y} d y
\end{aligned}
$$

using integration by parts. Apply this to (3.2) to get

$$
V(H)=e^{-r T} e^{2 \mu a / \sigma^{2}} \int_{\log \left(K / S_{0} e^{2 a}\right)}^{\infty}\left(1-\Phi\left(\frac{y-\mu T}{\sigma \sqrt{T}}\right)\right) S_{0} e^{2 a} e^{y} d y
$$

and make the substitution $x=(y+2 a) /(\sigma \sqrt{T})$ to get (3.3).

The price of a down-and-out call can be obtained by subtracting the down-and-in price from the standard Black-Scholes price. For a comprehensive treatment of the other cases and of connections among the various prices, see Rich (1994) and Rubinstein and Reiner (1991).

We now write $V\left(S_{0} e^{a}\right)$ for $V(H)$ and calculate the derivative of the continuous option price with respect to $a$ :

PROPOSITION 3.2. For a down-and-in call,

$$
\begin{aligned}
\frac{\partial}{\partial a} V\left(S_{0} e^{a}\right)= & e^{-r T}\left(\frac{2 \mu \sqrt{T}}{\sigma}\right) e^{2 \mu a / \sigma^{2}} \int_{\left[\log \left(K / S_{0}\right)\right] / \sigma \sqrt{T}}^{\infty} \\
& \times\left(1-\Phi\left(x-\frac{\mu \sqrt{T}}{\sigma}-\frac{2 a}{\sigma \sqrt{T}}\right)\right) S_{0} e^{x \sigma \sqrt{T}} d x \\
& +2 e^{-r T} e^{2 \mu a / \sigma^{2}} \int_{\left[\log \left(K / S_{0}\right)\right] / \sigma \sqrt{T}}^{\infty} \varphi\left(x-\frac{\mu \sqrt{T}}{\sigma}-\frac{2 a}{\sigma \sqrt{T}}\right) \\
& \times S_{0} e^{x \sigma \sqrt{T}} d x .
\end{aligned}
$$

Proof. From (3.2) we get

$$
\frac{\partial}{\partial a} V\left(S_{0} e^{a}\right)=\frac{2 \mu}{\sigma^{2}} e^{2 \mu a / \sigma^{2}} \operatorname{BSC}\left(S_{0} e^{2 a}\right)+e^{2 \mu a / \sigma^{2}} \cdot 2 S_{0} e^{2 a} \Delta\left(S_{0} e^{2 a}\right)
$$

where $\Delta(\cdot)$ is the Black-Scholes delta as a function of the initial asset price with $r, T, \sigma$, and $K$ held fixed. Substituting $\left(2 \mu / \sigma^{2}\right)$ times (3.3) for the first term yields the first term of 
(3.4). To get the second term in (3.4) we use (3.2) and (3.3) again and differentiate to get

$$
\begin{aligned}
2 S_{0} e^{2 a} \Delta\left(S_{0} e^{2 a}\right)=e^{-r T} \frac{\partial}{\partial a}[ & \int_{\left[\log \left(K / S_{0}\right)\right] / \sigma \sqrt{T}}^{\infty}\left(1-\Phi\left(x-\frac{\mu \sqrt{T}}{\sigma}-\frac{2 a}{\sigma \sqrt{T}}\right)\right) \\
& \left.\quad \times S_{0} e^{x \sigma \sqrt{T}} \sigma \sqrt{T} d x\right] \\
= & e^{-r T}\left(\frac{2}{\sigma \sqrt{T}}\right) \int_{\left[\log \left(K / S_{0}\right)\right] / \sigma \sqrt{T}}^{\infty} \varphi\left(x-\frac{\mu \sqrt{T}}{\sigma}-\frac{2 a}{\sigma \sqrt{T}}\right) \\
& \times S_{0} e^{x \sigma \sqrt{T}} \sigma \sqrt{T} d x .
\end{aligned}
$$

A simple justification of this interchange of derivative and integral first makes the change of variable $y=x-(2 a / \sigma \sqrt{T})$. This moves $a$ out of the argument of $\Phi$ and into the lower limit of integration.

Combining Propositions 3.1 and 3.2, we obtain formulas for the option price and its derivative in expressions of the form $V\left(S_{0} e^{a+\epsilon}\right)=V\left(S_{0} e^{a}\right)+\epsilon \partial_{a} V\left(S_{0} e^{a}\right)+o(\epsilon)$, where $\partial_{a}$ is short for $\partial / \partial a$.

\section{MAIN STEPS OF THE PROOF}

In this section, we give the main steps of the proof of Theorem 1.1. Most of the technical details of intermediate steps are relegated to appendices.

\subsection{The Discrete Price}

As in Section 1, we use $\tilde{S}_{n}$ to denote the asset price at the $n^{\text {th }}$ monitoring instant, $n T / m$. We use the representation

$$
\begin{aligned}
\tilde{S}_{n} & =S_{0} \exp \left\{\mu n \Delta t+\sigma \sqrt{\Delta t} \sum_{i=1}^{n} Z_{i}\right\} \\
& =S_{0} \exp \left\{\tilde{W}_{n} \sigma \sqrt{\Delta t}\right\}
\end{aligned}
$$

where the $Z_{i}$ are independent standard normal random variables,

$$
\tilde{W}_{n}=\sum_{i=1}^{n}\left(Z_{i}+\frac{\mu}{\sigma} \sqrt{\Delta t}\right),
$$

$\mu=r-\frac{1}{2} \sigma^{2}$, and $\Delta t=T / m$. Also, we let

$$
\begin{aligned}
\tilde{\tau} & =\inf \left\{n \geq 1: \tilde{S}_{n} \leq H\right\} \\
& =\inf \left\{n \geq 1: \tilde{W}_{n} \leq a /(\sigma \sqrt{\Delta t})\right\}
\end{aligned}
$$


where, as before, $a=\log \left(H / S_{0}\right)<0$. The option price is

$$
V_{m}=e^{-r T} E\left[\left(\tilde{S}_{m}-K\right)^{+} ; \tilde{\tau}<m\right]=e^{-r T} E\left[\left(S_{0} e^{\tilde{W}_{m} \sigma \sqrt{\Delta t}}-K\right)^{+} ; \tilde{\tau}<m\right] .
$$

This expectation is an integral with respect to the density in $x$ of the distribution $P(\tilde{\tau}<$ $\left.m, \tilde{W}_{m} \leq x\right)$. Integration by parts and a change of variables yields the following:

\section{LEMMA 4.1. With the notation above}

$$
V_{m}=e^{-r T} \int_{\left[\log \left(K / S_{0}\right)\right] / \sigma \sqrt{T}}^{\infty} P\left(\tilde{\tau}<m, \tilde{W}_{m} \geq y \sqrt{m}\right) \sigma \sqrt{T} S_{0} e^{y \sigma \sqrt{T}} d y .
$$

Our approach to proving Theorem 1.1 will be to approximate the integrand $P(\tilde{\tau}<$ $\left.m, \tilde{W}_{m} \geq y \sqrt{m}\right)$ for $y \geq \log \left(K / S_{0}\right) / \sigma \sqrt{T}$ to terms of order $m^{-3 / 2}$; evaluate the coefficients on the terms of order $m^{-1 / 2}$ and integrate them, as required by Lemma 4.1; show that the higher-order terms are $o\left(m^{-1 / 2}\right)$, even after integration; and finally identify the resulting expressions with continuous formulas from Section 3.

\subsection{Approximation of the Probability}

To approximate the integrand in Lemma 4.1, we first generalize the setting slightly. We use notation consistent with Siegmund and Yuh (1982), on which Theorem 4.1 below builds. We work with a family of probability measures $\left\{P_{\theta}, \theta \in \mathbb{R}\right\}$; under $P_{\theta}$, the random variables $\left\{X_{1}, X_{2}, \ldots\right\}$ are independent and normally distributed with mean $\theta$ and unit variance. Define

$$
U_{n}=\sum_{i=1}^{n} X_{i}
$$

under $P_{\theta}$, the random walk $U_{n}$ thus has $\operatorname{drift} \theta$. Let $\theta_{1}=\xi / \sqrt{m}$ and $\theta_{0}=-\xi / \sqrt{m}$, with $\xi \geq 0$ a finite constant. Define

$$
\tau^{\prime}=\inf \left\{n \geq 1: U_{n} \geq b\right\}
$$

where $b=\zeta \sqrt{m}$ for some $\zeta<\xi$. To make the correspondence with the notation of Section 4.1, set

$$
\xi=-\frac{\mu \sqrt{T}}{\sigma} \quad \text { and } \quad \zeta=-\frac{a}{\sigma \sqrt{T}}>0
$$

Then

$$
P\left(\tilde{\tau}<m, \tilde{W}_{m} \geq y \sqrt{m}\right)=P_{\theta_{1}}\left(\tau^{\prime}<m, U_{m} \leq-y \sqrt{m}\right)
$$

with $y \geq a /(\sigma \sqrt{T})=-\zeta$, as in Lemma 4.1. By the symmetry of the normal distribution, the case $\xi<0$ (corresponding to $\mu>0$ ) follows from an analysis of $\xi \geq 0$. 
THEOREM 4.1. Let $R_{m}=U_{\tau^{\prime}}-b$, and define

$$
\begin{aligned}
& A\left(\tau^{\prime}, y\right)=\Phi\left(-\frac{\zeta+y}{\sqrt{1-\tau^{\prime} / m}}-\xi \sqrt{1-\tau^{\prime} / m}\right) \\
& B\left(\tau^{\prime}, y\right)=\frac{1}{\sqrt{1-\tau^{\prime} / m}} \varphi\left(\frac{\zeta+y}{\sqrt{1-\tau^{\prime} / m}}+\xi \sqrt{1-\tau^{\prime} / m}\right) .
\end{aligned}
$$

Then for $y>-\zeta$,

$$
\begin{aligned}
P_{\theta_{1}}\left(\tau^{\prime}<m,\right. & \left.U_{m}<-y \sqrt{m}\right)=e^{2 \xi \zeta}(1-\Phi(2 \zeta+y+\xi)) \\
& +\frac{2 \xi}{\sqrt{m}} E_{\theta_{1}}\left[R_{m} A\left(\tau^{\prime}, y\right) ; \tau^{\prime}<m\right]-\frac{2}{\sqrt{m}} E_{\theta_{1}}\left[R_{m} B\left(\tau^{\prime}, y\right) ; \tau^{\prime}<m\right] \\
& -\frac{1}{m} E_{\theta_{1}}\left[C \cdot 4 \xi^{2} R_{m}^{2} A\left(\tau^{\prime}, y\right) ; \tau^{\prime}<m\right]+\frac{2 \xi}{m} E_{\theta_{1}}\left[R_{m}^{2} B\left(\tau^{\prime}, y\right) ; \tau^{\prime}<m\right] \\
& -\frac{1}{m \sqrt{m}} E_{\theta_{1}}\left[D \cdot 4 \xi^{2} R_{m}^{2} B\left(\tau^{\prime}, y\right) ; \tau^{\prime}<m\right] \\
& -\frac{1}{m} E_{\theta_{1}}\left[e^{-2 \xi R_{m} / \sqrt{m}} R_{m}^{2}\left(1-\tau^{\prime} / m\right)^{-1} \cdot \frac{1}{2} \varphi^{\prime}\left(\eta_{1}\right) ; \tau^{\prime}<m\right] \\
& +\frac{1}{m} E_{\theta_{1}}\left[R_{m}^{2}\left(1-\tau^{\prime} / m\right)^{-1} \cdot \frac{1}{2} \varphi^{\prime}\left(\eta_{2}\right) ; \tau^{\prime}<m\right],
\end{aligned}
$$

where $|C| \leq 1,|D| \leq 1$,

$$
\eta_{1} \in\left[\frac{\zeta+y}{\sqrt{1-\tau^{\prime} / m}}+\xi \sqrt{1-\tau^{\prime} / m}-\frac{R_{m}}{\sqrt{1-\tau^{\prime} / m}}, \frac{\zeta+y}{\sqrt{1-\tau^{\prime} / m}}+\xi \sqrt{1-\tau^{\prime} / m}\right]
$$

and

$$
\eta_{2} \in\left[\frac{\zeta+y}{\sqrt{1-\tau^{\prime} / m}}+\xi \sqrt{1-\tau^{\prime} / m}, \frac{\zeta+y}{\sqrt{1-\tau^{\prime} / m}}+\xi \sqrt{1-\tau^{\prime} / m}+\frac{R_{m}}{\sqrt{1-\tau^{\prime} / m}}\right]
$$

Proof. See Appendix A.

\subsection{Limits of the Coefficients}

In light of Lemma 4.1, integrating equation (4.2) in Theorem 4.1 yields the discrete price $V_{m}$. To approximate the discrete price, we evaluate the integral of each of the terms in the right-hand side of (4.2) as $m \rightarrow \infty$. An essential tool is the following result:

LEMMA 4.2. There is a probability distribution $B_{R}$ on $[0, \infty)$, such that for any $t, y \geq 0$,

$$
P_{\theta_{1}}\left(\tau^{\prime} / m \leq t, R_{m} \leq y\right) \rightarrow G(t ; \xi, \zeta) B_{R}(y)
$$


as $m \rightarrow \infty$, where

$$
G(t ; \xi, \zeta)=1-\Phi\left(\zeta T^{-1 / 2}-\xi \sqrt{T}\right)+e^{2 \xi \zeta} \Phi\left(-\zeta T^{-1 / 2}-\xi \sqrt{T}\right)
$$

Moreover, $\int y d B_{R}(y)=\beta$, with $\beta$ as defined in Theorem 1.1.

REMARK. $G(t ; \xi, \zeta)$ is the probability that a Wiener process with drift $\xi$ and variance parameter 1 reaches $\zeta$ by time $t$.

Proof. From Lemma 10.11 of Siegmund (1985) we have

$$
P_{\theta_{1}}\left(\tau^{\prime} \leq \zeta^{2} m t, R_{m} \leq y\right) \rightarrow G(t ; \xi, \zeta) B_{R}(y)
$$

Hence,

$$
\begin{aligned}
P_{\theta_{1}}\left(\tau^{\prime} / m \leq t, R_{m} \leq y\right) & \rightarrow G\left(t / \zeta^{2} ; \xi \zeta, 1\right) B_{R}(y) \\
& =G(t ; \xi, \zeta) B_{R}(y),
\end{aligned}
$$

as can be seen from the formula for $G$. Theorem 10.55 of Siegmund (1985) gives a general expression for the mean of $B_{R}$. Specializing to the case of normal increments and comparing with Chernoff (1965) we find that this mean is the $\beta$ defined in Theorem 1.1.

We interpret this result as stating that $\tau^{\prime} / m$ and $R_{m}$ are asymptotically independent, and that the distributions of $\tau^{\prime} / m$ and $R_{m}$ can be approximated by $G$ and $B_{R}$ respectively. So, to approximate the expectations in Theorem 4.1, we factor them into products of expectations involving just $R_{m}$ and just $\tau^{\prime}$, then evaluate them under the limiting marginal distributions indicated by Lemma 4.2. To approximate integrals over $y$ of these expectations (as required by Lemma 4.1), we integrate the approximations to the expectations. The next result justifies this process.

Proposition 4.1. Denote by $g$ the density of the distribution $G$. Let $\beta_{1}=\beta$ and let $\beta_{2}$ denote the second moment of $B_{R}$. Then,

$$
\begin{aligned}
& \lim _{m \rightarrow \infty} \int_{\left[\log \left(K / S_{0}\right)\right] / \sigma \sqrt{T}}^{\infty} E_{\theta_{1}}\left[R_{m}^{\ell} A\left(\tau^{\prime}, y\right) ; \tau^{\prime}<m\right] e^{y \sigma \sqrt{T}} d y \\
& \quad=\beta_{\ell} \int_{\left[\log \left(K / S_{0}\right)\right] / \sigma \sqrt{T}}^{\infty} \int_{0}^{1} \Phi\left(-\frac{\zeta+y}{\sqrt{1-t}}-\xi \sqrt{1-t}\right) g(t) d t e^{y \sigma \sqrt{T}} d y, \quad \ell=1,2,
\end{aligned}
$$

and

$$
\begin{aligned}
\lim _{m \rightarrow \infty} & \int_{\left[\log \left(K / S_{0}\right)\right] / \sigma \sqrt{T}}^{\infty} E_{\theta_{1}}\left[R_{m}^{\ell} B\left(\tau^{\prime}, y\right) ; \tau^{\prime}<m\right] e^{y \sigma \sqrt{T}} d y \\
= & \beta_{\ell} \int_{\left[\log \left(K / S_{0}\right)\right] / \sigma \sqrt{T}}^{\infty} \int_{0}^{1} \frac{1}{\sqrt{1-t}} \varphi\left(\frac{\zeta+y}{\sqrt{1-t}}+\xi \sqrt{1-t}\right) \\
& \times g(t) d t e^{y \sigma \sqrt{T}} d y, \quad \ell=1,2 .
\end{aligned}
$$




\section{Proof. See Appendix B.}

To evaluate the limits in Proposition 4.1, we need the following identities:

LEMMA 4.3.

$$
\int_{0}^{1} \Phi\left(-\frac{x}{\sqrt{1-t}}-\xi \sqrt{1-t}\right) g(t) d t=e^{2 \xi \zeta} \Phi(-x-\zeta-\xi)
$$

$$
\int_{0}^{1} \frac{1}{\sqrt{1-t}} \varphi\left(\frac{x}{\sqrt{1-t}}+\xi \sqrt{1-t}\right) g(t) d t=e^{2 \xi \zeta} \varphi(x+\zeta+\xi)
$$

Proof. Both identities can in principle be verified algebraically using the fact that

$$
g(t)=\frac{\zeta}{\sqrt{2 \pi}} t^{-3 / 2} \exp \left\{\xi \zeta-\frac{1}{2}\left(\frac{\zeta^{2}}{t+\xi^{2} t}\right)\right\}, \quad t>0
$$

An alternative probabilistic argument notes that both sides of (4.5) are equal to $P\left(\tau^{(\xi)}<\right.$ $\left.1, W_{1}^{(\xi)}<\zeta-x\right)$, where $W^{(\xi)}$ is a unit-variance Wiener process with drift $\xi$, and $\tau^{(\xi)}$ is the first time $W^{(\xi)}$ reaches $\zeta$. For the right side use equation (3.14) of Siegmund (1985) and for the left side condition on $\tau$. Both sides of (4.6) yield the corresponding density at $\zeta-x$.

Using Proposition 4.1 and Lemma 4.3, we can handle integrals of the first five expectations on the right side of equation (4.2) in Theorem 4.1. To dispense with integrals of the last two expectations there, we need the following:

PROPOSITION 4.2. For $i=1,2$,

$$
\limsup _{m \rightarrow \infty} E_{\theta_{1}}\left[\int_{\left[\log \left(K / S_{0}\right)\right] / \sigma \sqrt{T}}^{\infty} \varphi^{\prime}\left(\eta_{i}\right) \frac{e^{y \sigma \sqrt{T}}}{\sqrt{1-\tau^{\prime} / m}} d y ; \tau^{\prime}<m\right]<\infty
$$

where $\eta_{1}, \eta_{2}$ are as in Theorem 4.1.

\section{Proof. See Appendix C.}

\subsection{Comparison with the Continuous Price}

Consider the expression given for the discrete price in Lemma 4.1. For the integrand appearing there, substitute the expression in (4.2) using the parameters in (4.1). Theorem 4.1 applies because for any $y \geq \log \left(K / S_{0}\right) / \sigma \sqrt{T}$ we have $y>\log \left(H / S_{0}\right) / \sigma \sqrt{T}=-\zeta$. The 
result of the substitution is of the form

$$
\begin{aligned}
e^{r T} V_{m}\left(S_{0} e^{a}\right)= & \int_{\left[\log \left(K / S_{0}\right)\right] / \sigma \sqrt{T}}^{\infty} e^{2 \mu a / \sigma^{2}}\left(1-\Phi\left(y-\frac{2 a}{\sigma \sqrt{T}}-\frac{\mu \sqrt{T}}{\sigma}\right)\right) \\
& \times \sigma \sqrt{T} S_{0} e^{y \sigma \sqrt{T}} d y \\
& +\frac{1}{\sqrt{m}} \int_{\left[\log \left(K / S_{0}\right)\right] / \sigma \sqrt{T}}^{\infty} 2 E_{\theta_{1}}\left[R_{m}\left(\xi A\left(\tau^{\prime}, u\right)-B\left(\tau^{\prime}, y\right)\right) ; \tau^{\prime}<m\right] \\
& \times \sigma \sqrt{T} S_{0} e^{y \sigma \sqrt{T}} d y \\
& +\frac{1}{m} \int \cdots d y \\
& +\frac{1}{m \sqrt{m}} \int \cdots d y .
\end{aligned}
$$

Now apply (3.3) to the first integral, and invoke the boundedness of the limits in Proposition 4.1 for $\ell=2$ and the boundedness proved in Proposition 4.2 to write this as

$$
\begin{aligned}
e^{r T} V_{m}\left(S_{0} e^{a}\right)= & e^{r T} V\left(S_{0} e^{a}\right) \\
& +\frac{1}{\sqrt{m}} \int_{\left[\log \left(K / S_{0}\right)\right] / \sigma \sqrt{T}}^{\infty} 2 E_{\theta_{1}}\left[R_{m}\left(\xi A\left(\tau^{\prime}, u\right)-B\left(\tau^{\prime}, y\right)\right) ; \tau^{\prime}<m\right] \\
& \times \sigma \sqrt{T} S_{0} e^{y \sigma \sqrt{T}} d y+o\left(\frac{1}{\sqrt{m}}\right) .
\end{aligned}
$$

We may replace the remaining integral with its limit as $m \rightarrow \infty$ and preserve the validity of this expression. Using Proposition 4.1 with $\ell=1$ and then Lemma 4.3, we thus obtain

$$
\begin{aligned}
V_{m}\left(S_{0} e^{a}\right)= & V\left(S_{0} e^{a}\right)+\frac{2 \xi \beta \sigma \sqrt{T}}{\sqrt{m}} e^{-r T} \int_{\left[\log \left(K / S_{0}\right)\right] / \sigma \sqrt{T}}^{\infty} e^{2 \xi \zeta} \Phi(-2 \zeta-y-\xi) S_{0} e^{y \sigma \sqrt{T}} d y \\
& -\frac{2 \beta \sigma \sqrt{T}}{\sqrt{m}} e^{-r T} \int_{\left[\log \left(K / S_{0}\right)\right] / \sigma \sqrt{T}}^{\infty} e^{2 \xi \zeta} \varphi(2 \zeta+y+\xi) S_{0} e^{y \sigma \sqrt{T}} d y+o\left(\frac{1}{\sqrt{m}}\right) .
\end{aligned}
$$

Substituting for $\xi$ and $\zeta$ as in (4.1), we get

$$
\begin{aligned}
& V_{m}\left(S_{0} e^{a}\right)= \\
& V\left(S_{0} e^{a}\right)-\frac{\beta \sigma \sqrt{T}}{\sqrt{m}} \frac{2 \mu \sqrt{T}}{\sigma} e^{-r T} \int_{\left[\log \left(K / S_{0}\right)\right] / \sigma \sqrt{T}}^{\infty} e^{2 \mu a / \sigma^{2}} \\
& \times\left(1-\Phi\left(y-\frac{\mu \sqrt{T}}{\sigma}-\frac{2 a}{\sigma \sqrt{T}}\right)\right) S_{0} e^{y \sigma \sqrt{T}} d y \\
& -\frac{\beta \sigma \sqrt{T}}{\sqrt{m}} 2 e^{-r T} e^{2 \mu a / \sigma^{2}} \int_{\left[\log \left(K / S_{0}\right)\right] / \sigma \sqrt{T}}^{\infty} \varphi\left(y-\frac{\mu \sqrt{T}}{\sigma}-\frac{2 a}{\sigma \sqrt{T}}\right) S_{0} e^{y \sigma \sqrt{T}} d y \\
& +o\left(\frac{1}{\sqrt{m}}\right) .
\end{aligned}
$$


Comparing this with Proposition 3.2, we may rewrite it as

$$
V_{m}\left(S_{0} e^{a}\right)=V\left(S_{0} e^{a}\right)-\frac{\beta \sigma \sqrt{T}}{\sqrt{m}} \partial_{a} V\left(S_{0} e^{a}\right)+o\left(\frac{1}{\sqrt{m}}\right)
$$

which is to say that

$$
V_{m}\left(S_{0} e^{a}\right)=V\left(S_{0} e^{a-\beta \sigma \sqrt{\Delta t}}\right)+o\left(\frac{1}{\sqrt{m}}\right)
$$

concluding the proof of Theorem 1.1.

Notice that if we simply omit the integral from the expression in Lemma 4.1, we obtain the price of a discretely monitored binary barrier option. Omitting the integral from (3.3) produces the price of the corresponding continuously monitored barrier option. The steps carried out in proving Theorem 1.1 for the integrals apply equally well—indeed, with much less effort—for the integrands. Thus, we have the following corollary.

COROLLARY 4.1. Theorem 1.1 holds for binary barrier options as well.

\section{APPENDIX A: PROOF OF THEOREM 4.1}

Directly from Siegmund and Yuh (1982, p. 244), we have

$$
\begin{aligned}
P_{\theta_{1}}\left(\tau^{\prime}<m, U_{m}<-y \sqrt{m}\right)=e^{\left(\theta_{1}-\theta_{0}\right) b} P_{\theta_{0}}\left(U_{m} \geq(2 \zeta+y) \sqrt{m}\right) \\
\quad-E_{\theta_{1}}\left[e^{-\left(\theta_{1}-\theta_{0}\right) R_{m}}\right. \\
\left.\quad \times\left\{1-F_{\theta_{0}, m-\tau^{\prime}}\left(\sqrt{m}(y+\zeta)-R_{m}\right)-F_{\theta_{1}, m-\tau^{\prime}}\left(-\sqrt{m}(y+\zeta)-R_{m}\right)\right\} ; \tau^{\prime}<m\right],
\end{aligned}
$$

where $F_{\theta, n}$ is the distribution of $U_{n}$ under $P_{\theta}$. Let us write this as

$$
P_{\theta_{1}}\left(\tau^{\prime}<m, U_{m}<-y \sqrt{m}\right)=\mathbf{X}-E_{\theta_{1}}\left[\mathbf{Y} ; \tau^{\prime}<m\right] .
$$

Then

$$
\begin{aligned}
\mathbf{X} & =e^{2 \xi \zeta}\left(1-\Phi\left(\frac{(2 \zeta+y) \sqrt{m}-\theta_{0} m}{\sqrt{m}}\right)\right) \\
& =e^{2 \xi \zeta}(1-\Phi(2 \zeta+y+\xi)),
\end{aligned}
$$

which is exactly the first term on the right side of equation (4.2) in Theorem 4.1.

Next we analyze $\mathbf{Y}$. By the definition of $U_{n}$, each $F_{\theta, n}$ is a normal distribution with mean $n \theta$ and variance $n$. Thus,

$$
\begin{aligned}
\mathbf{Y}= & e^{-2 \xi R_{m} / \sqrt{m}}\left(1-\Phi\left(\frac{(y+\zeta) \sqrt{m}-R_{m}+\xi\left(m-\tau^{\prime}\right) / \sqrt{m}}{\sqrt{m-\tau^{\prime}}}\right)\right) \\
& -\Phi\left(\frac{-(y+\zeta) \sqrt{m}-R_{m}-\xi\left(m-\tau^{\prime}\right) / \sqrt{m}}{\sqrt{m-\tau^{\prime}}}\right) .
\end{aligned}
$$


Taylor expansion now gives

$$
\begin{aligned}
\mathbf{Y}=e^{-2 \xi R_{m} / \sqrt{m}}\left[1-\Phi\left(\frac{y+\zeta}{\sqrt{1-\tau^{\prime} / m}}+\xi \sqrt{1-\tau^{\prime} / m}\right)\right. \\
\left.\quad+\frac{R_{m}}{\sqrt{m-\tau^{\prime}}} \varphi\left(\frac{y+\zeta}{\sqrt{1-\tau^{\prime} / m}}+\xi \sqrt{1-\tau^{\prime} / m}\right)+\frac{1}{2} \frac{R_{m}^{2}}{m-\tau^{\prime}} \varphi^{\prime}\left(\eta_{1}\right)\right] \\
-\quad\left[\Phi\left(-\frac{y+\zeta}{\sqrt{1-\tau^{\prime} / m}}-\xi \sqrt{1-\tau^{\prime} / m}\right)\right. \\
\quad-\frac{R_{m}}{\sqrt{m-\tau^{\prime}}} \varphi\left(-\frac{y+\zeta}{\sqrt{1-\tau^{\prime} / m}}-\xi \sqrt{1-\tau^{\prime} / m}\right) \\
\left.\quad+\frac{1}{2} \frac{R_{m}^{2}}{m-\tau^{\prime}} \varphi^{\prime}\left(\eta_{2}\right)\right],
\end{aligned}
$$

for some $\eta_{1}, \eta_{2}$ as stated in the theorem. Because $\Phi(-x)=1-\Phi(x)$ and $\varphi(-x)=\varphi(x)$, this becomes

$$
\begin{aligned}
\mathbf{Y}= & \left(e^{-2 \xi R_{m} / \sqrt{m}}-1\right) \Phi\left(-\frac{y+\zeta}{\sqrt{1-\tau^{\prime} / m}}-\xi \sqrt{1-\tau^{\prime} / m}\right) \\
& +\left(e^{-2 \xi R_{m} / \sqrt{m}}+1\right) \frac{R_{m}}{\sqrt{m-\tau^{\prime}}} \varphi\left(\frac{y+\zeta}{\sqrt{1-\tau^{\prime} / m}}+\xi \sqrt{1-\tau^{\prime} / m}\right) \\
& +e^{-2 \xi R_{m} / \sqrt{m}} \frac{R_{m}^{2}}{m-\tau^{\prime}} \frac{1}{2} \varphi^{\prime}\left(\eta_{1}\right)-\frac{R_{m}^{2}}{m-\tau^{\prime}} \frac{1}{2} \varphi^{\prime}\left(\eta_{2}\right) .
\end{aligned}
$$

For any $x \geq 0$ there is a $c$ with $|c| \leq 1$, such that $e^{-x}=1-x+c x^{2}$. We may thus introduce $C$ and $D$ as stated in the theorem to get

$$
\begin{aligned}
\mathbf{Y}= & \left(-2 \xi R_{m} / \sqrt{m}\right) \Phi\left(-\frac{y+\zeta}{\sqrt{1-\tau^{\prime} / m}}-\xi \sqrt{1-\tau^{\prime} / m}\right) \\
& +C \cdot 4 \xi^{2} \frac{R_{m}^{2}}{m} \Phi\left(-\frac{y+\zeta}{\sqrt{1-\tau^{\prime} / m}}-\xi \sqrt{1-\tau^{\prime} / m}\right) \\
& +\left(2-2 \xi R_{m} / \sqrt{m}\right) \frac{R_{m}}{\sqrt{m-\tau^{\prime}}} \varphi\left(\frac{y+\zeta}{\sqrt{1-\tau^{\prime} / m}}+\xi \sqrt{1-\tau^{\prime} / m}\right) \\
& +D \cdot 4 \xi^{2} \frac{R_{m}^{2}}{m} \frac{R_{m}}{\sqrt{m-\tau^{\prime}}} \varphi\left(\frac{y+\zeta}{\sqrt{1-\tau^{\prime} / m}}+\xi \sqrt{1-\tau^{\prime} / m}\right) \\
& +e^{-2 \xi R_{m} / \sqrt{m}} \frac{R_{m}^{2}}{m-\tau^{\prime}} \frac{1}{2} \varphi^{\prime}\left(\eta_{1}\right)-\frac{R_{m}^{2}}{m-\tau^{\prime}} \frac{1}{2} \varphi^{\prime}\left(\eta_{2}\right) .
\end{aligned}
$$


With $A\left(\tau^{\prime}, y\right)$ and $B\left(\tau^{\prime}, y\right)$ as defined in the theorem, this becomes

$$
\begin{aligned}
\mathbf{Y}= & \left(-2 \xi R_{m} / \sqrt{m}\right) A\left(\tau^{\prime}, y\right)+C \cdot 4 \xi^{2} \frac{R_{m}^{2}}{m} A\left(\tau^{\prime}, y\right) \\
& +\left(2-2 \xi R_{m} / \sqrt{m}\right) \frac{R_{m}}{\sqrt{m}} B\left(\tau^{\prime}, y\right)+D \cdot 4 \xi^{2} \frac{R_{m}^{3}}{m \sqrt{m}} B\left(\tau^{\prime}, y\right) \\
& +e^{-2 \xi R_{m} / \sqrt{m}} \frac{R_{m}^{2}}{m-\tau^{\prime}} \frac{1}{2} \varphi^{\prime}\left(\eta_{1}\right)-\frac{R_{m}^{2}}{m-\tau^{\prime}} \frac{1}{2} \varphi^{\prime}\left(\eta_{2}\right)
\end{aligned}
$$

Grouping terms according to their denominators, we get

$$
\begin{aligned}
\mathbf{Y}= & \frac{-2 \xi R_{m}}{\sqrt{m}} A\left(\tau^{\prime}, y\right)+\frac{2 R_{m}}{\sqrt{m}} B\left(\tau^{\prime}, y\right) \\
& +\frac{1}{m} C \cdot 4 \xi^{2} R_{m}^{2} A\left(\tau^{\prime}, y\right)-\frac{1}{m} 2 \xi R_{m}^{2} B\left(\tau^{\prime}, y\right) \\
& +\frac{1}{m \sqrt{m}} D \cdot 4 \xi^{2} R_{m}^{2} B\left(\tau^{\prime}, y\right) \\
& +e^{-2 \xi R_{m} / \sqrt{m}} \frac{R_{m}^{2}}{m-\tau^{\prime}} \frac{1}{2} \varphi^{\prime}\left(\eta_{1}\right)-\frac{R_{m}^{2}}{m-\tau^{\prime}} \frac{1}{2} \varphi^{\prime}\left(\eta_{2}\right) .
\end{aligned}
$$

Via (A.1), this concludes the proof of the theorem.

\section{APPENDIX B: UNIFORM INTEGRABILITY}

The main objective of this appendix is to prove Proposition 4.1. We begin by recording two useful facts. From Lemma 10.11 of Siegmund (1985) we get convergence of all moments of the overshoot to moments of $B_{R}$ :

$$
\lim _{m \rightarrow \infty} E_{\theta_{1}}\left[R_{m}^{q}\right]=\int y^{q} d B_{R}(y), \quad \text { for all } q>0
$$

In particular, $E_{\theta_{1}}\left[R_{m}\right] \rightarrow \beta$. We will also use the easily verified identity

$$
\int_{b}^{\infty} e^{c y} \varphi(u+y) d y=e^{c(c+2 u) / 2} \Phi(-b+c+u) .
$$

We now prove

LEMMA B.1. There is a finite constant $M$ such that for all $m$

$$
\sup _{0 \leq t<m} \int_{\left[\log \left(K / S_{0}\right)\right] / \sigma \sqrt{T}}^{\infty} B(t, y) e^{y \sigma \sqrt{T}} d y \leq M .
$$


Proof. Using first the definition of $B(t, y)$ and then (B.2), we get

$$
\begin{aligned}
& \int_{\left[\log \left(K / S_{0}\right)\right] / \sigma \sqrt{T}}^{\infty} B(t, y) e^{y \sigma \sqrt{T}} d y \\
& =\int_{\left[\log \left(K / S_{0}\right)\right] / \sigma \sqrt{T}}^{\infty} \varphi\left(\frac{\zeta+y}{\sqrt{1-t / m}}+\xi \sqrt{1-t / m}\right) e^{y \sigma \sqrt{T}} \frac{d y}{\sqrt{1-t / m}} \\
& =\exp \left(\frac{1}{2} \sigma \sqrt{T} \sqrt{1-t / m}\left[\sigma \sqrt{T} \sqrt{1-t / m}+2 \xi \sqrt{1-t / m}+\frac{2 \zeta}{\sqrt{1-t / m}}\right]\right) \\
& \quad \times \Phi\left(-\frac{\log \left(K / S_{0}\right)}{\sigma \sqrt{T} \sqrt{1-t / m}}+\sigma \sqrt{T} \sqrt{1-t / m}+\xi \sqrt{1-t / m}+\frac{\zeta}{\sqrt{1-t / m}}\right),
\end{aligned}
$$

and this is bounded for $0 \leq t<m$.

We proceed with the proof of Proposition 4.1, first considering (4.4). Because all quantities inside the integral and expectation on the left side of (4.4) are nonnegative, by Tonelli's Theorem we may interchange the integral and expectation to get

$$
\begin{aligned}
& \int_{\left[\log \left(K / S_{0}\right)\right] / \sigma \sqrt{T}}^{\infty} E_{\theta_{1}}\left[R_{m}^{\ell} B\left(\tau^{\prime}, y\right) ; \tau^{\prime}<m\right] e^{y \sigma \sqrt{T}} d y \\
& \quad=E_{\theta_{1}}\left[R_{m}^{\ell} \int_{\left[\log \left(K / S_{0}\right)\right] / \sigma \sqrt{T}}^{\infty} B\left(\tau^{\prime}, y\right) e^{y \sigma \sqrt{T}} d y ; \tau^{\prime}<m\right] .
\end{aligned}
$$

By Lemma B.1, the integral inside the expectation is uniformly bounded. But then by (B.1) the entire expression inside the expectation is uniformly integrable. To evaluate the limit as $m \rightarrow \infty$, we may therefore integrate with respect to the limiting distribution to get

$$
\beta_{\ell} \int_{0}^{1} \int_{\left[\log \left(K / S_{0}\right)\right] / \sigma \sqrt{T}}^{\infty} \frac{1}{\sqrt{1-t}} \varphi\left(\frac{\zeta+y}{\sqrt{1-t}}+\xi \sqrt{1-t}\right) e^{y \sigma \sqrt{T}} d y g(t) d t
$$

Again interchanging the order of integration yields (4.4).

The proof of (4.3) is similar. Just as in Lemma B.1, we integrate to verify that

$$
\int_{\left[\log \left(K / S_{0}\right)\right] / \sigma \sqrt{T}}^{\infty} A(t, y) e^{y \sigma \sqrt{T}} d y
$$

is uniformly bounded for $0 \leq t<m$. This proves uniform integrability which, together with two applications of Tonelli's Theorem yields (4.3).

\section{APPENDIX C: PROOF OF PROPOSITION 4.2}

We begin with the following bound on the integral inside the expectation: 
LEMMA C.1. There are finite constants $M_{1}, M_{2}$, and $M_{3}$ for which

$$
\sup _{0 \leq t<m}\left|\int_{\left[\log \left(K / S_{0}\right)\right] / \sigma \sqrt{T}}^{\infty} \varphi^{\prime}\left(\eta_{i}\right) \frac{e^{y \sigma \sqrt{T}}}{\sqrt{1-t / m}} d y\right| \leq M_{1}+M_{2} e^{M_{3} R_{m} / \sqrt{m}}
$$

for $i=1,2$.

Proof. For any $v>0$, integration by parts shows that

$$
\int_{b}^{\infty} e^{c y} \varphi^{\prime}(u+v y) d y=-\frac{1}{v} e^{c b} \varphi(u+v b)-\frac{c}{v^{2}} \exp \left(\frac{1}{2} \frac{c}{v}\left(\frac{c}{v}+2 u\right)\right) \Phi\left(-v b+\frac{c}{v}+u\right) .
$$

Apply this identity to the integral in the lemma by setting $b=\log \left(K / S_{0}\right) /(\sigma \sqrt{T}), c=$ $\sigma \sqrt{T}, v=1 / \sqrt{1-t / m}$, and $u=\zeta(1-t / m)^{-1 / 2}+\xi \sqrt{1-t / m}+d_{i}, i=1$, 2, with

$$
\begin{aligned}
& d_{1} \in\left[-\frac{R_{m}}{\sqrt{m} \sqrt{1-t / m}}, 0\right] \\
& d_{2} \in\left[0, \frac{R_{m}}{\sqrt{m} \sqrt{1-t / m}}\right] .
\end{aligned}
$$

This results in a bound of the form in the lemma.

The proof of Proposition 4.2 will be complete once we verify that

$$
\lim \sup E_{\theta_{1}}\left[\exp \left(M_{3} R_{m} / \sqrt{m}\right)\right]
$$

is finite as $m \rightarrow \infty$. For this we need

LEMMA C.2. There is a finite constant $M_{4}$ such that $P_{\theta_{1}}\left(R_{m}>u\right) \leq M_{4} e^{-u}$, for all $m$ and all $u \geq 0$.

Proof. Since $1-\Phi(x) \leq \frac{1}{x} e^{-x^{2} / 2} \leq e^{-x}$ for all $x>0$, by the argument of Example 2.2 on p. 19 of Woodroofe (1982) we have

$$
\begin{aligned}
P_{\theta_{1}}\left(R_{m}>u\right) & =\sum_{n=1}^{\infty} P_{\theta_{1}}\left(\tau^{\prime} \geq n, U_{n}>b+u\right) \\
& \leq \sum_{n=1}^{\infty} E_{\theta_{1}}\left[e^{-\left(b+u-U_{n-1}\right)} ; \tau^{\prime} \geq n\right] \\
& =e^{-u} \sum_{n=1}^{\infty} E_{\theta_{1}}\left[e^{-\left(b-U_{n-1}\right)} ; \tau^{\prime} \geq n\right] \\
& \equiv M_{4} e^{-u} .
\end{aligned}
$$


It follows now that

$$
E_{\theta_{1}}\left[e^{M_{3} R_{m} / \sqrt{m}}\right] \leq M_{4} \int_{0}^{\infty} e^{M_{3} r / \sqrt{m}} e^{-r} d r
$$

which remains bounded as $m \rightarrow \infty$.

\section{REFERENCES}

AitSahliA, F. (1995): "Optimal Stopping and Weak Convergence Methods for Some Problems in Financial Economics," Doctoral dissertation, Department of Operations Research, Stanford University, Stanford, CA.

ASMUSSEN, S. (1989): "Risk Theory in a Markovian Environment," Scand. Actuarial J., 16, 69-100.

BLACK, F., and M. SCHOLES (1973): "The Pricing of Options and Corporate Liabilities," J. Political Economy, 81, 637-659.

BoYLE, P. P., and S. H. LAU (1994): "Bumping Up Against the Barrier with the Binomial Method," J. Derivatives, 2, 6-14.

Broadie, M., P. Glasserman, and S. Kou (1996): "Connecting Discrete and Continuous PathDependent Options," working paper, Columbia Business School, New York, to appear in Finance and Stochastics.

Chance, D. M. (1994): "The Pricing and Hedging of Limited Exercise Caps and Spreads," $J$. Financial Res., 17, 561-584.

Chernoff, H. (1965): "Sequential Tests for the Mean of a Normal Distribution IV," Ann. Math. Statist., 36, 55-68.

Chernoff, H., and A. J. PetKaU (1986): "Numerical Solutions for Bayes Sequential Decision Problems," SIAM J. Sci. Stat. Comput., 7, 46-59.

Derivatives Week (1995a): "Discrete Path-Dependent Options," April 10, 7.

Derivatives Week (1995b): "Liquidity Standards to be Drawn up for Barrier Options," May 29, 2.

FlESAKER, B. (1992): "The Design and Valuation of Capped Stock Index Options," working paper, Department of Finance, University of Illinois, Champaign, IL.

HeYNEN, R. C., and H. M. KAT (1994a): “Crossing Barriers," RISK, 7 (June), 46-49. Correction (1995), RISK, 8, (March) 18.

Heynen, R. C., and H. M. Kat (1994b): "Partial Barrier Options," J. Financial Engineering, 3 (September/December), 253-274.

Karatzas, I., and S. ShreVe (1991): Brownian Motion and Stochastic Calculus. New York: Springer-Verlag.

KAT, H., and L. VERDONK (1995): “Tree Surgery,” RISK, 8 (February), 53-56.

Kunitomo, N., and M. IKedA (1992): "Pricing Options with Curved Boundaries," Math. Finance, 2, 275-298.

Merton, R. C. (1973): “Theory of Rational Option Pricing,” Bell J. Econ. Mgmt. Sci., 4, 141-183.

Rich, D. (1994): “The Mathematical Foundations of Barrier Option Pricing Theory,” Adv. Futures Opt. Res., 7, 267-312.

Rich, D. (1996): "The Valuation of Black-Scholes Options Subject to Intertemporal Default Risk," Rev. Derivatives Res., 1, 25-59.

RUBINSTEIN, M., and E. REINER (1991): "Breaking Down the Barriers," RISK, 4 (September), 28-35.

SIEGMUND, D. (1979): “Corrected Diffusion Approximations in Certain Random Walk Problems," Adv. Appl. Probab., 11, 701-719. 
SIEGMund, D. (1985): Sequential Analysis: Tests and Confidence Intervals. New York: SpringerVerlag.

SIEGMUND, D., and Y.-S. YUH (1982): "Brownian Approximations for First Passage Probabilities," Z. Wahrsch. verw. Gebiete, 59, 239-248.

Woodroofe, M. (1982): Nonlinear Renewal Theory in Sequential Analysis. Philadelphia: Society for Industrial and Applied Mathematics. 